\title{
Beam instabilities in a magnetized pair plasma
}

\author{
M A XIM L Y UTIKOV* \\ Theoretical Astrophysics, California Institute of Technology, Pasadena, CA 91125, USA
}

(Received 22 July 1998 and in revised form 6 April 1999)

\begin{abstract}
Beam instabilities in the strongly magnetized electron-positron plasma of a pulsar magnetosphere are considered. We analyse the resonance conditions and estimate the growth rates of the Cherenkov and cyclotron instabilities of the ordinary $(\mathrm{O})$, extraordinary $(\mathrm{X})$ and Alfvén modes in two limiting regimes: kinetic and hydrodynamic. The importance of the different instabilities as a source of coherent pulsar radiation generation is then estimated, taking into account the angular dependence of the growth rates and the limitations on the length of the coherent wave-particle interaction imposed by the curvature of the magnetic field lines. We conclude that in the pulsar magnetosphere, Cherenkov-type instabilities occur in the hydrodynamic regime, while cyclotron-type instabilities occur in the kinetic regime. We argue that electromagnetic cyclotron-type instabilities on the $\mathrm{X}, \mathrm{O}$ and probably Alfvén waves are more likely to develop in the pulsar magnetosphere.
\end{abstract}

\section{Introduction}

At present, the most promising theories of pulsar radio emission generation are based on the plasma emission model, in which the high-brightness radio emission is generated by plasma instabilities developing in an outflowing one-dimensional electron-positron plasma penetrated by a highly relativistic electron or positron beam (Sturrock 1960; Goldreich and Julian 1969; Melrose 1995). To find the instability responsible for the generation of pulsar radio emission, it is essential to know the dispersion relations of the normal modes of the medium and take into account the evolution of the modes as they propagate outwards in the pulsar magnetosphere.

In this paper, we resort to similar distributions of the pair plasma with equal densities, and neglect the curvature of the magnetic field lines. These approximations are justified if the radius of curvature of field lines in the particle's rest frame, $R_{B} / \gamma_{p}^{2}$, is much less than the Larmor radius $r_{L}=c / \omega_{B}: R_{B} / \gamma_{p}^{2} \ll r_{L}$ (here $\gamma_{p}$ is the average streaming energy of plasma particles in the pulsar frame).

We consider wave excitation in a strongly magnetized pair plasma in the approximation of straight magnetic field lines, thus omitting an important Cherenkov-drift resonance (Lyutikov et al. 1999a,b) and other effects of inhomogeneity (Goldreich and Keeley 1971; Beskin et al. 1983; Asseo et al. 1983; Asseo 1995). The electromagnetic Cherenkov-drift instability, which may be responsible for the generation of cone-type emission in pulsars, occurs in the kinetic regime on the high-frequency vacuum-like $\mathrm{O}$ and $\mathrm{X}$ waves. It has the same advantages as the electromagnetic cyclotron instabilities considered in this paper.

${ }^{\star}$ Present address: Canadian Institute for Theoretical Astrophysics, McLennan Labs, $60 \mathrm{St}$ George Street, Toronto, Canada M5S 3H8. 
Owing to the limitations of space, we have had to omit numerous details of the work, which can be found in Lyutikov (1998).

The main conclusions of our work are the following. For the chosen parameters of the magnetospheric plasma, the Cherenkov-type electrostatic beam instabilities develop in the hydrodynamic regime, while cyclotron-type electromagnetic instabilities develop in the kinetic regime. Electrostatic beam instabilities in the pulsar plasma are generally weaker than electromagnetic instabilities. In addition, Cherenkov instabilities have the largest growth rate near the stellar surface, where the Cherenkov resonance can occur only on the Alfvén mode. However, this mode cannot escape to infinity, even though it has an electromagnetic component. Another factor that limits the development of Cherenkov-type instabilities is that they grow within a much narrower angle than cyclotron instabilities. In a curved magnetic, field this results in a shorter length of coherent wave-particle interaction.

The relative weakness of electrostatic instabilities as compared with electromagnetic instabilities is an unusual characteristics of strongly relativistic beams. The reason for this is that, for the particles in the primary beam, which contribute to the development of the instability, the effective parallel mass $m_{\mathrm{eff} \|}=\gamma_{b}^{3} \mathrm{~m} \approx 10^{21} \mathrm{~m}$. This suppresses the development of the electrostatic instabilities. In contrast, the effective transverse mass $m_{\text {eff } \perp}=\gamma_{b} m$ is less affected by the large parallel momentum. The electromagnetic instabilities are less suppressed by the large streaming momenta. Thus the relativistic velocities and one-dimensionality of the distribution function result in strong suppression of electrostatic instabilities as compared with electromagnetic instabilities.

The calculations described here provided a basis for the model of pulsar radio emission presented in Lyutikov et al. (1999a).

\section{Plasma parameters}

To a large extent, possible mechanisms for the generation of pulsar radio emission are predicated on the choice of parameters of the plasma flow generated by a rotating neutron star. At present, we know only the general features of the distribution function of the particles in a pulsar magnetosphere (Tademaru 1973; Arons 1981; Daugherty and Harding 1983). It is believed to comprise (see Fig. 1)

(i) a highly relativistic primary beam with Lorentz factor $\gamma_{b} \approx 10^{7}$ and density equal to the Goldreich-Julian density $n_{G J}$;

(ii) a secondary electron-positron plasma with a bulk streaming Lorentz factor $\gamma_{p} \approx 10-1000$, a similar scatter in energy $T_{p} \approx \gamma_{p}$ and a density $n_{p}$ much larger than the beam density: $n_{p} \approx \lambda n_{G J}=10^{3}-10^{6} n_{G J}$;

(iii) a tail with energy up to $\gamma_{t}=10^{4}-10^{5}$.

We shall normalize the density of the pair plasma to the Goldreich-Julian density.

$$
n_{\alpha}=\lambda n_{G J}=10^{3}-10^{6} n_{G J}, \quad \omega_{p}^{2}=\lambda \omega_{b}^{2}=2 \lambda \omega_{B} \Omega
$$

(the subscript $\alpha$ in (2.1) refers to the electrons and positrons of the bulk plasma).

Another relation between the parameters of the plasma and those of the beam comes from the energy argument that the primary particles stop producing pairs when the energy in the pair plasma becomes equal to the energy in the primary 


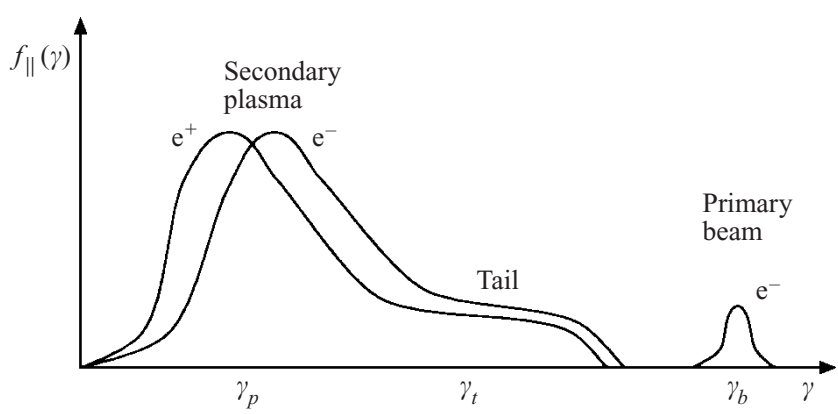

Figure 1. Distribution function for a one-dimensional electron-positron in a plasma of pulsar magnetosphere.

beam:

$$
2\langle\gamma\rangle_{ \pm} n_{ \pm}=\gamma_{b}^{\prime} n_{G J}, \quad \text { at the pair formation front }
$$

where $\langle\gamma\rangle_{ \pm}$and $n_{ \pm}$are the initial average energies and densities of pair plasma, $\gamma_{b}^{\prime}$ is the energy of the beam in the pulsar frame (quantities measured in the pulsar frame will be indicated with a prime ${ }^{\prime}$ ) and $n_{G J}$ is the density of the beam. Initial densities, temperatures and velocities of the plasma components are equal.

The uncertainty in the physics of the pair-formation front forces us to allow for a broad range of plasma parameters. Accordingly, the growth rates of the particular instabilities can vary considerably, depending on the assumed parameters. Numerical estimates will be given for a typical pulsar with period $P=0.5 \mathrm{~s}$ (light-cylinder radius $R_{l l}=2.4 \times 10^{9} \mathrm{~cm}$ ), surface magnetic field $B=10^{12} \mathrm{G}$ and primary-beam Lorentz factor $\gamma_{b}=2 \times 10^{7}$ (see e.g. Arons 1983). These assumptions and (2.2) reduce the number of free parameters to two: plasma temperature and bulk streaming energy $\gamma_{p}$ (or temperature and multiplicity factor $\lambda$ ). Consequently, we shall consider two separate cases of a cold and a relativistically hot plasma. For numerical estimates, we shall use the following fiducial numbers: $\gamma_{p}=100, \lambda=10^{5}, T_{p} \ll 1$ for the cold plasma, and $\gamma_{p}=100, \lambda=10^{4}, T_{p} \approx 10$ for the relativistically hot plasma $\left(T_{p}\right.$ is the invariant temperature of the plasma in units of $m c^{2}$ ). The radial dependence of the parameters is assumed to follow the dipole geometry of the magnetic field.

\section{Response tensor for a one-dimensional plasma in straight magnetic field}

In the limits of applicability of our simplifying assumptions, the components of the dielectric tensor are (Lyutikov et al. 1999a)

$$
\begin{aligned}
\epsilon_{x x} & =1-\frac{1}{2} \sum_{\alpha} \frac{\omega_{p \alpha}^{2}}{\omega^{2}} \int \frac{d p_{z}}{\gamma}\left(\omega-k_{z} v_{z}\right) A_{\alpha}^{+} f_{\alpha}=\epsilon_{y y} \\
\epsilon_{z z} & =1-\sum_{\alpha} \omega_{p \alpha}^{2} \int \frac{d p_{z}}{\gamma^{3}} \frac{f_{\alpha}}{\left(\Omega_{\alpha}^{0}\right)^{2}}-\sum_{\alpha} \frac{\omega_{p \alpha}^{2}}{\omega^{2}} \int \frac{d p_{z}}{\gamma} f_{\alpha} \frac{\left(k_{x}^{2}+k_{y}^{2}\right) v_{z}^{2}}{\Omega_{\alpha}^{+} \Omega_{\alpha}^{-}} \\
\epsilon_{x y} & =-\frac{i}{2} \sum_{\alpha} \frac{\omega_{p \alpha}^{2}}{\omega^{2}} \int \frac{d p_{z}}{\gamma}\left(\omega-k_{z} v_{z}\right) A_{\alpha}^{-} f_{\alpha}=-\epsilon_{y x} \\
\epsilon_{x z} & =\frac{1}{2} \sum_{\alpha} \frac{\omega_{p \alpha}^{2}}{\omega^{2}} \int \frac{d p_{z}}{\gamma} v_{z}\left(k_{x} A_{\alpha}^{+}+i k_{y} A_{\alpha}^{-}\right) f_{\alpha}=\epsilon_{z x}^{*}
\end{aligned}
$$


68

$$
\epsilon_{y z}=-\frac{1}{2} \sum_{\alpha} \frac{\omega_{p \alpha}^{2}}{\omega^{2}} \int \frac{d p_{z}}{\gamma} \frac{v_{z}}{c}\left(k_{y} A_{\alpha}^{+}-i k_{x} c A_{\alpha}^{-}\right) f_{\alpha}=\epsilon_{z y}^{*} .
$$

Here

$$
\begin{aligned}
A_{\alpha}^{+} & =\frac{1}{\Omega_{\alpha}^{+}}+\frac{1}{\Omega_{\alpha}^{-}}, \quad A_{\alpha}^{-}=\frac{1}{\Omega_{\alpha}^{-}}-\frac{1}{\Omega_{\alpha}^{+}}, \\
\Omega_{\alpha}^{ \pm} & =\omega-k_{z} v_{z} \pm \omega_{B} \gamma^{-1}, \quad \Omega_{\alpha}^{0}=\omega-k_{z} v_{z}
\end{aligned}
$$

$f_{\alpha}$ is the one-dimensional distribution function of component $\alpha, v_{z}$ is the velocity along the local magnetic field, $\gamma$ is the Lorentz factor of a particle, $k_{x}, k_{z}$ and $k_{y}$ are the corresponding components of the wave vector, and the magnetic field is directed along the $z$ axis; the asterisk * denotes the complex conjugate.

The normal modes satisfy the dispersion relation

$$
\operatorname{Det}\left|\Lambda_{\alpha \beta}\right|=0, \quad \text { where } \Lambda_{\alpha \beta}=k_{\alpha} k_{\beta}-k^{2} c^{2} \delta_{\alpha \beta}+\omega^{2} \epsilon_{\alpha \beta}(\omega, \mathbf{k})
$$

and $\epsilon_{\alpha \beta}(\omega, \mathbf{k})$ is the dielectric tensor of the medium.

\section{Waves in a pair plasma}

In the pulsar magnetosphere, the waves that may be important for the generation of the observed radio emission have frequencies much less than the gyrofrequency. In what follows, we shall use the low-frequency approximation, in which all the relevant frequencies are much less than the gyrofrequency. In the cold plasma in its rest frame, this implies that $\omega \ll \omega_{B}$.

Here we give explicitly only a few dispersion relations (see also Arons and Barnard 1986). A detailed analysis of the properties of the normal modes is given elsewhere (Lyutikov 1998).

\subsection{Waves in a cold plasma in its rest frame}

In a one-dimensional plasma, the dispersion equation (3.3) factorizes, giving three wave branches: a transverse $\mathrm{X}$ mode with electric vector perpendicular to the $(\mathbf{k}, \mathbf{B})$ plane, and two coupled longitudinal-transverse $\mathrm{O}$ and Alfvén branches with the electric vector in the (k,B) plane (see Fig. 2 ). The $\mathrm{X}$ wave is a subluminal transverse electromagnetic wave with a dispersion relation

$$
\omega_{X}^{2}=k^{2} c^{2}\left(1-\frac{2 \omega_{p}^{2}}{\omega_{B}^{2}}\right)=k^{2} v_{A}^{2}, \quad \omega \ll \omega_{B},
$$

where $v_{A}$ is the Alfvén velocity in a strongly magnetized plasma. The Alfvén branch is always subluminal, while the $\mathrm{O}$ mode is superluminal at small wave vectors and subluminal at large wave vectors. The crossover point, where the $\mathrm{O}$ mode becomes luminal, is

$$
\omega_{0}^{2}=k_{0}^{2} c^{2}=2 \omega_{p}^{2}+\omega_{B}^{2} \sin ^{2} \theta
$$

The large- and small-wave-vector asymptotic solutions for the $\mathrm{O}$ and Alfvén branches are

$$
\omega^{2}= \begin{cases}k^{2} c^{2}\left(1-\frac{2 \omega_{p}^{2} \cos ^{2} \theta}{\omega_{B}^{2}}\right)+2 \omega_{p}^{2} \sin ^{2} \theta & \text { (O wave) } \\ 2 \omega_{p}^{2} \cos ^{2} \theta\left(1-\frac{2 \omega_{p}^{2} \sin ^{2} \theta}{k^{2} c^{2}}-\frac{2 \omega_{p}^{2} \sin ^{2} \theta}{\omega_{B}^{2}}\right) & \text { (Alfvén wave) }\end{cases}
$$




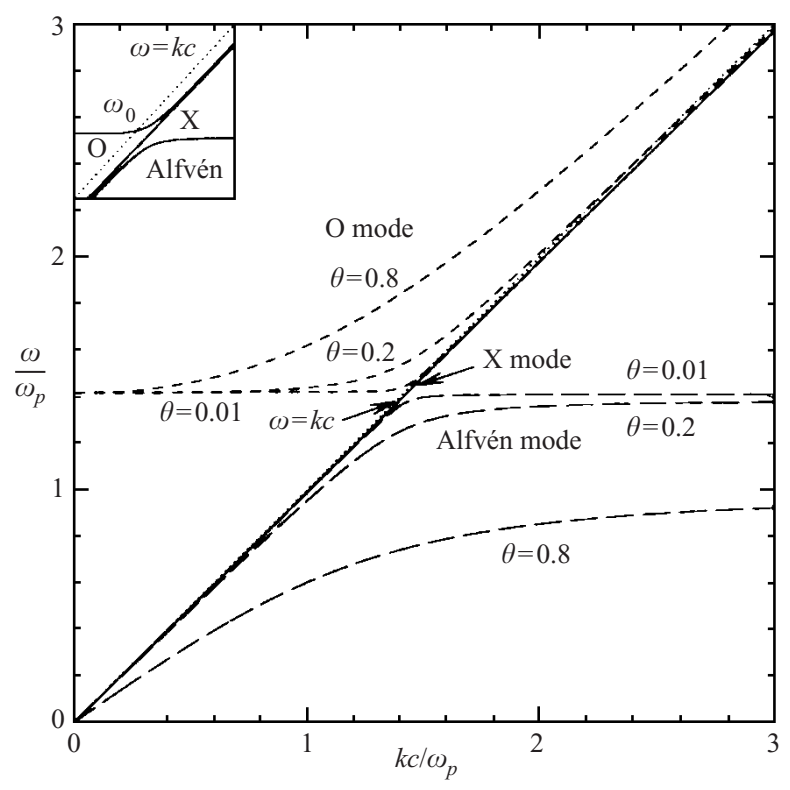

Figure 2. Dispersion curves for the waves in a cold electron-positron plasma in the plasma frame in the limit $\omega_{p} \ll \omega_{B}$. There are three modes represented by dashed (O mode), solid ( $\mathrm{X}$ mode) and long-dashed (Alfvén mode) lines. The dotted line represents the vacuum dispersion relation. For exactly parallel propagation, the dispersion curves for the $\mathrm{O}$ mode and the Alfvén mode intersect. The insert in the upper left corner shows the region near the crossover point $\omega_{0}$.

if $k c \gg \omega_{p}$, and

$$
\omega^{2}= \begin{cases}2 \omega_{p}^{2}+k^{2} c^{2}\left(1-\frac{k^{2} c^{2} \cos ^{2} \theta}{\omega_{p}^{2}}\right) \sin ^{2} \theta & (\text { O wave }) \\ k^{2} c^{2} \cos ^{2} \theta\left(1-\frac{2 \omega_{p}^{2}}{\omega_{B}^{2}}-\frac{k^{2} c^{2} \sin ^{2} \theta}{2 \omega_{p}^{2}}\right) & \text { (Alfvén wave) }\end{cases}
$$

if $k c \ll \omega_{p}$.

The polarization vectors for these normal modes are

$\mathbf{e}_{X}=(0,1,0)$,

$\mathbf{e}_{O}= \begin{cases}\left(\cos \theta\left(1-\frac{2 \omega_{p}^{2} \sin ^{2} \theta}{\omega^{2}}\right), 0,\left(1+\frac{2 \omega_{p}^{2} \cos ^{2} \theta}{\omega^{2}}\right) \sin \theta\right)+O\left(\frac{\omega_{p}^{2}}{k^{2} c^{2}}\right) & \left(k c \gg \omega_{p}\right), \\ \left(\frac{-k^{2} \sin 2 \theta}{4 \omega_{p}^{2}}, 0,1\right)+O\left(\frac{k^{2} c^{2}}{\omega_{p}^{2}}\right) & \left(k c \ll \omega_{p}\right),\end{cases}$

$\mathbf{e}_{O}= \begin{cases}\left(\frac{\omega_{B}^{2} \theta}{\omega_{0}^{2}}, 0,-1\right) & \left(\theta \ll \frac{2 \omega_{p}^{2}}{\omega_{B}^{2}}, \omega \approx \omega_{0}\right), \\ \left(1,0,-\frac{\omega_{0}^{2} \operatorname{cosec} \theta \sec \theta}{\omega_{B}^{2}}\right) & \left(\theta \gg \frac{2 \omega_{p}^{2}}{\omega_{B}^{2}}, \omega \approx \omega_{0}\right),\end{cases}$ 


$$
\mathbf{e}_{A}= \begin{cases}\left(\left(1+\frac{2 \omega_{p}^{2} \cos ^{2} \theta}{k^{2}}\right) \sin \theta, 0, \cos \theta\left(1-\frac{2 \omega_{p}^{2} \sin ^{2} \theta}{k^{2}}\right)\right)+O\left(\frac{\omega_{p}^{2}}{k^{2}}\right) & \left(k c \gg \omega_{p}\right), \\ \left(1,0, \frac{\omega^{2} \tan \theta}{2 \omega_{p}^{2}}\right)+O\left(\frac{k^{2} c^{2}}{\omega_{p}^{2}}\right) & \left(k c \ll \omega_{p}\right) .\end{cases}
$$

\subsection{Waves in a relativistically hot pair plasma}

We have investigated the effects of the thermal motion of plasma particles on the dispersion relation of normal modes for two generic distribution functions: waterbag and relativistic Maxwellian (see also Silin 1960; Tsytovich and Kaplan 1972; Godfrey and Shanahan 1974; Melrose 1982; Suvorov and Chugunov 1975; Volokitin et al. 1985; Arons and Barnard 1986; Zank and Greaves 1995; Lyutikov 1998). The thermal motion of plasma particles considerably affects the dispersion of the Alfvén mode at frequencies $\omega \geqslant \omega_{p}$ and the dispersion of the $\mathrm{O}$ mode at frequencies $\omega \approx \omega_{p}$. Another important quantitative modification is in the dispersion relations of the $\mathrm{X}$ mode and $\mathrm{O}$ mode at large frequencies $\omega \geqslant \omega_{p}$. An important factor in the excitation of these modes is the difference between the phase speed and the speed of light. This difference is roughly proportional to $\left\langle 1 / \gamma^{3}\right\rangle$. It is considerably decreased owing to the bulk streaming of the plasma. In a relativistically hot streaming plasma, there are more particles with low Lorentz factors, which contribute to $\left\langle 1 / \gamma^{3}\right\rangle$, than in a cold plasma streaming with the same average velocity. So, for a given streaming velocity, a relativistically hot plasma has a larger $\left\langle 1 / \gamma^{3}\right\rangle$ and a larger growth rate.

The dispersion relation for the $\mathrm{X}$ mode in a hot plasma is

$$
\omega^{2}=k^{2} c^{2}\left(1-\frac{\omega_{p}^{2}}{\omega_{B}^{2}} T_{p}\left(1+\beta_{T}^{2} \cos ^{2} \theta\right)\right),
$$

where $\beta_{T}=\left(1-1 / T^{2}\right)^{1 / 2}$.

It is possible to obtain an asymptotic expansion of the dispersion relations of the Alfvén and $\mathrm{O}$ modes in the limits of very small and very large wave vectors. In the limit $k c \gg T_{p}^{1 / 2} \omega_{p}$, we have

$\omega^{2}=\left\{\begin{array}{l}c^{2} k^{2} \beta_{T}^{2} \cos ^{2} \theta\left(1-\frac{2 \omega_{p}^{2}}{c^{2} T_{p}^{3} k^{2} \beta_{T}^{2}\left(-1+\beta_{T}^{2} \cos ^{2} \theta\right)}\right) \quad \text { (Alfvén wave) }, \\ c^{2} k^{2}\left(1-\frac{\omega_{p}^{2}}{\omega_{B}^{2}} T_{p}\left(1+\beta_{T}^{2} \cos ^{2} \theta\right)\right)\left(1-\frac{2 \omega_{p}^{2} \sin ^{2} \theta}{c^{2} T_{p} k^{2}\left(-1+\beta_{T}^{2} \cos ^{2} \theta\right)}\right)(\mathrm{O} \text { wave }),\end{array}\right.$

while in the opposite limit $k c \ll T_{p}^{1 / 2} \omega_{p}$

$\omega^{2}= \begin{cases}c^{2} k^{2} \cos ^{2} \theta\left(1-\frac{\omega_{p}^{2}}{\omega_{B}^{2}} T_{p}\left(1+\beta_{T}^{2} \cos ^{2} \theta\right)\right)\left(1-\frac{c^{2} k^{2} \sin ^{2} \theta}{2 T_{p} \omega_{p}^{2}}\right) & \text { (Alfvén wave) } \\ \frac{2 \omega_{p}^{2}}{T_{p}}+c^{2} k^{2}\left(\beta_{T}^{2} \cos ^{2} \theta+\sin ^{2} \theta\right) & \text { (O wave) }\end{cases}$

The $\mathrm{X}$ mode is always superluminal and the Alfvén mode is always subluminal. The $\mathrm{O}$ mode is superluminal for small small vectors $k c \ll T_{p}{ }^{1 / 2} \omega_{p}$, and may become subluminal for very small angles of propagation $\theta \ll T_{p}{ }^{1 / 2} \omega_{p} / \omega_{B}$. 
The crossover point (where the phase speed of the $\mathrm{O}$ mode become equal to the speed of light) is now

$$
\omega_{0}^{(h) 2}=k_{0}^{2} c^{2} \approx 2 T_{p} \omega_{p}^{2}+\omega_{B}^{2} \sin ^{2} \theta .
$$

The polarization vectors for the $\mathrm{O}$ and Alfvén modes are

$$
\begin{aligned}
& \mathbf{e}_{O}^{(h)}= \begin{cases}\left(\cos \theta\left(1-\frac{2 T_{p} \omega_{p}^{2} \sin ^{2} \theta}{c^{2} k^{2}}\right), 0,-\left(1+\frac{2 T_{p} \omega_{p}^{2} \cos ^{2} \theta}{c^{2} k^{2}}\right) \sin \theta\right)\left(k c \gg \omega_{p}\right), \\
\left(\frac{\omega_{B}^{2} \theta}{\omega_{0}^{2}}, 0,-1\right) \quad\left(\theta \ll \frac{2 T_{p} \omega_{p}^{2}}{\omega_{B}^{2}}, \omega=\omega_{0}^{(h)}\right), \\
\left(1,0,-\frac{\omega_{0}^{2}}{\sin \theta \cos \theta \omega_{B}^{2}}\right) \quad\left(\theta \gg \frac{2 T_{p} \omega_{p}^{2}}{\omega_{B}^{2}}, \omega=\omega_{0}^{(h)}\right),\end{cases} \\
& \mathbf{e}_{A}^{(h)}=\left(1,0, \frac{\omega^{2} \tan \theta}{2 T_{p} \omega_{p}^{2}}\right) \quad\left(k c \ll \omega_{p}\right) .
\end{aligned}
$$

\section{Hydrodynamic and kinetic instabilities}

For the beam-plasma system the dielectric tensor $\epsilon_{\alpha \beta}(\omega, \mathbf{k})$ may be represented as the sum of the contributions from the plasma and the beam:

$$
\epsilon_{\alpha \beta}(\omega, \mathbf{k})=\delta_{\alpha \beta}+\frac{4 \pi c}{\omega} \sigma_{\alpha \beta}^{\text {plasma }}+\frac{4 \pi c}{\omega} \sigma_{\alpha \beta}^{\text {beam }},
$$

where $\sigma_{\alpha \beta}^{\text {plasma }}$ and $\sigma_{\alpha \beta}^{\text {beam }}$ are the conductivity tensors of the plasma and the beam.

Two separate cases may be distinguished here, depending on whether the complex part of the beam contribution to the dispersion relations is zero or non-zero. This corresponds to the two types of instabilities: hydrodynamic and kinetic. In hydrodynamic instabilities, all the particles of the beam resonate with the normal mode of the plasma. This requires that the growth rate of the instability be greater than the intrinsic bandwidth of the growing waves:

$$
|\mathbf{k} \cdot \delta \mathbf{v}| \ll \operatorname{Im}[\Delta(\mathbf{k})],
$$

where $\mathbf{k}$ is the resonant wave vector and $\delta \mathbf{v}$ is the scatter in the velocity of the beam particles.

For a one-dimensional plasma, the condition of hydrodynamic approximation is

$$
k_{\|} c \frac{\Delta \gamma}{\gamma^{3}}+\frac{s \omega_{B} \Delta \gamma}{\gamma^{2}} \ll \Gamma
$$

where $\Delta \gamma$ is the spread in Lorentz factors, and $s=0, \pm 1, \ldots$ In the kinetic regime, this inequality is reversed.

For an instability to be important as a possible source of coherent emission generation, its growth rate, evaluated in the pulsar frame, should be much larger than the pulsar rotation frequency $\Omega$ :

$$
\frac{\Gamma}{\gamma_{p} \Omega} \gg 1 .
$$

Another, more stringent, requirement on the growth rate comes from its angular 


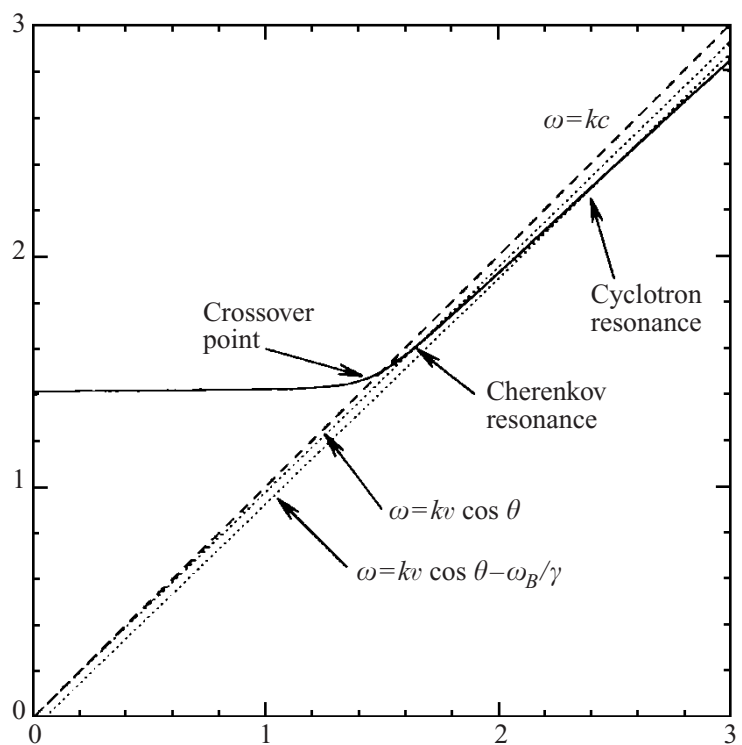

Figure 3. Resonances on the $\mathrm{O}$ mode in a cold plasma for $\mu>1$.

dependence. The emitting plasma propagates in a curved magnetic field. If an instability has a considerable growth inside a characteristic angle $\delta \theta^{\prime}$ then the growth length should be larger than $\delta \theta R_{c}$, where $R_{c}$ is the curvature of the magnetic field. In the plasma frame, this requirement is

$$
\Gamma \gg \frac{c \gamma_{p}^{2}}{R_{c} \delta \theta}
$$

\section{Cold pair plasma: resonances}

In a cold plasma, the resonant interaction between the fast particles and the plasma may be considered as the interaction of waves in the plasma with waves in the beam. The interaction is strongest when the dispersion relations of the waves intersect. Consequently, we are looking for the possible resonances between the waves in the plasma and the waves in the beam (Fig. 3):

$$
\begin{aligned}
\omega & =v_{b} k_{z}, \\
\omega & =v_{b} k \cos \theta \pm \frac{\omega_{B}}{\gamma_{b}} .
\end{aligned}
$$

As we shall see in Section 8, the resonant interaction of the plasma waves with the Cherenkov waves in the beam (6.1) is described by a cubic equation for the frequency shift, which always has complex-conjugate solutions. This implies that the Cherenkov resonant interaction of the waves in the beam and those in the plasma is always unstable.

In contrast, the frequency shift due to the cyclotron interaction of the waves in the beam and in the plasma (6.2) is described by a quadratic equation, which has two real solutions for the plus sign in (6.2) and two complex solutions for the minus sign. Thus only the minus sign in (6.2) will contribute to the instability growth rate. The resonance $(6.2)$ with the minus sign is called anomalous Doppler 
resonance. This corresponding instability may be considered as the interaction of a negative-energy wave in the beam with a positive-energy wave in plasma. Owing to the resonant coupling, the amplitudes of both waves grow exponentially.

Now let us consider the condition for the resonances (6.1) and (6.2) to occur. From the low-frequency asymptotics of the Alfvén waves (4.4), we infer that the possibility of Cherenkov excitation of the Alfvén waves depends on the parameter

$$
\mu=\frac{2 \gamma_{b} \omega_{p}}{\omega_{B}}=5 \times 10^{-3}\left(\frac{r}{R_{N S}}\right)^{3 / 2}=\left\{\begin{array}{cc}
<1 & \left(\frac{r}{R_{N S}}<43\right) \\
>1 & \left(\frac{r}{R_{N S}}>43\right) .
\end{array}\right.
$$

If $\mu<1$ then Alfvén waves can be excited by Cherenkov resonance. $\dagger$ However, if $\mu>1$ then Alfvén waves cannot be excited by Cherenkov resonance; instead, resonance can occur for an $\mathrm{O}$ mode subject to the requirement of sufficiently small angles of propagation (Fig. 3).

At small radii $(\mu \ll 1)$, it is the Alfvén wave that is excited by the Cherenkov resonance, while for larger radii $(\mu \geqslant 1)$ it is the $O$ mode that can be excited by the Cherenkov resonance.

For parallel propagation (and only in this case), the parts of the $\mathrm{O}$ and Alfvén modes that have longitudinal polarization may be considered as forming a single plasma wave with a dispersion $\omega=2^{1 / 2} \omega_{p}$. In this particular case, the excitation of either the $\mathrm{O}$ or the Alfvén part of the longitudinal plasma mode is very similar. But as the waves propagate in curved magnetic field lines, the parts of the plasma mode corresponding to the $\mathrm{O}$ or the Alfvén wave will evolve differently, resulting in different observational characteristics of the emerging radiation.

We also note that the $\mathrm{X}$ wave cannot be excited by Cherenkov resonance. Although, formally intersection of the Cherenkov wave in the beam (6.1) with the dispersion relation of the $\mathrm{X}$ mode is possible for all frequencies if $\mu=1$, the transverse polarization of the $\mathrm{X}$ mode excludes resonant interaction with particles streaming along the magnetic field.

Cyclotron resonance on the $X$ modes occurs at $\omega_{\text {res }} \ll \omega_{B}$, provided that

$$
\frac{\omega_{p}^{2} \gamma_{b}}{\omega_{B}^{2}} \gg 1
$$

Using the fiducial plasma parameters of the cold plasma, we find

$$
\frac{\omega_{p}^{2} \gamma_{b}}{\omega_{B}^{2}}=\lambda \gamma_{b} \frac{2 \Omega}{\gamma_{p} \omega_{B}}=1.3 \times 10^{-10}\left(\frac{r}{R_{N S}}\right)^{3},
$$

which implies that the $\mathrm{X}$ mode can be excited by cyclotron resonance only in the outer parts of the magnetosphere for radii satisfying

$$
\frac{r_{\mathrm{res}}}{R_{N S}}>\left(\frac{\omega_{B}^{*} \gamma_{p}^{2}}{\lambda \gamma_{b}^{\prime} \Omega}\right)^{1 / 3} \approx 2 \times 10^{3} .
$$

The location of the cyclotron resonance on the $\mathrm{X}$ mode is quite sensitive to the

$\dagger$ In the case of a cold plasma, this may be considered as a sufficient condition for the Cherenkov excitation of Alfvén waves. In the case of a hot plasma, it is only a necessary condition (see below). 
choice of the bulk streaming energy. Comparing the resonant frequency (see Table 9 below) with the plasma frequency, we find

$$
\frac{\omega_{\mathrm{res}, \mathrm{X}, \mathrm{cycl}}}{\omega_{p}}=\frac{\omega_{B}^{3}}{\gamma_{b} \omega_{p}^{3}} \gg 1,
$$

which implies that the $\mathrm{X}$ mode is always excited with frequencies much larger than the plasma frequency.

\section{Hydrodynamic instabilities in a cold plasma}

\section{\%.1. Dielectric tensor for a cold beam-plasma system}

The dielectric tensor for a beam of density $n_{b}$ propagating with velocity $v_{b}$ along a magnetic field $B$ through a plasma of density $n$ can be found from the general expression (3.1) with zero drift velocity $u_{\alpha}=0$ and distribution function $f_{\alpha}\left(p_{z}\right)=$ $n_{p} \delta\left(p_{z}\right)+n_{b} \delta\left(p_{z}-p_{b}\right)\left(n_{b}\right.$ is the density of the beam and $p_{z}$ is the momentum of the beam particles):

$$
\begin{aligned}
& \epsilon_{x x}=1+\frac{2 \omega_{p}^{2}}{-\omega^{2}+\omega_{B}^{2}}-\frac{\omega_{b}^{2} \hat{\omega}^{2}}{\gamma_{b} \omega^{2} \tilde{\omega}^{2}}=\epsilon_{y y}, \\
& \epsilon_{x y}=\frac{-i \omega_{b}^{2} \omega_{B} \hat{\omega}}{\gamma_{b}^{2} \omega^{2} \tilde{\omega}^{2}}=-\epsilon_{y x}, \\
& \epsilon_{x z}=-\frac{k \omega_{b}^{2} \hat{\omega} v_{b} \sin \theta}{\gamma_{b} \omega^{2} \tilde{\omega}^{2}}=\epsilon_{z x}, \\
& \epsilon_{y z}=\frac{i k \omega_{b}^{2} \omega_{B} v_{b} \sin \theta}{\gamma_{b}^{2} \omega^{2} \tilde{\omega}^{2}}=-\epsilon_{z y}, \\
& \epsilon_{z z}=1-\frac{2 \omega_{p}^{2}}{\omega^{2}}-\frac{\omega_{b}^{2}}{\gamma_{b}^{3} \hat{\omega}^{2}}-\frac{k^{2} \omega_{b}^{2} v_{b}^{2} \sin ^{2} \theta}{\gamma_{b} \omega^{2} \tilde{\omega}^{2}},
\end{aligned}
$$

where

$$
\begin{aligned}
\hat{\omega} & =\omega-k v_{b} \cos \theta, \\
\tilde{\omega}^{2} & =\left(\omega-k v_{b} \cos \theta\right)^{2}-\frac{\omega_{B}^{2}}{\gamma_{b}^{2}}, \\
\gamma_{b} & =1-\left(\frac{v_{b}^{2}}{c^{2}}\right)^{-1 / 2}
\end{aligned}
$$

We shall always assume that the beam can be considered as a weak perturbation, so that we can employ the expansion procedure described in Sec. 5 .

\subsection{Parallel and perpedicular propagation}

In this subsection, we calculate the growth rates for the beam instabilities for the two particular cases of waves propagating along and perpendicular to the magnetic field. These will later serve as guidelines and estimates for the general case of oblique propagation.

For propagation along the magnetic field, the dispersion relation (3.3) with dielectric tensor (7.1) factorizes as

$$
-1+\frac{2 \omega_{p}^{2}}{\omega^{2}}+\frac{\omega_{b}^{2}}{\gamma_{b}^{3} \hat{\omega}^{2}}=0,
$$




$$
-1+n^{2}+\frac{2 \omega_{p}^{2}}{\omega^{2}-\omega_{B}^{2}}+\frac{\omega_{b}^{2} \hat{\omega}}{\gamma_{b} \omega^{2}\left( \pm \omega_{B} / \gamma_{b}+\hat{\omega}\right)}=0 .
$$

Equation (7.2) describes hydrodynamic excitation of longitudinal plasma waves. As discussed above, this may be a longitudinal part of the Alfvén or the $\mathrm{O}$ mode, depending on the parameters of the plasma. Equation (7.3) describes cyclotron excitation of the $\mathrm{O}$ and $\mathrm{X}$ modes. For parallel propagation, cyclotron excitation of the Alfvén wave does not occur.

To find the growth rate of Cherenkov excitation of plasma waves, we expand (7.2) in small frequency shifts $\omega_{l}=2^{1 / 2} \omega_{p}+\Delta=v_{b} k \cos \theta+\Delta$, and we find the imaginary part of the frequency shift:

$$
\operatorname{Im}(\Delta)=\frac{3^{1 / 2} \omega_{p}^{1 / 3} \omega_{b}^{2 / 3}}{2^{7 / 6} \gamma_{b}}=\frac{3^{1 / 2} \lambda^{1 / 6}\left(\Omega \omega_{B}\right)^{1 / 2}}{2^{2 / 3} \gamma_{b} \gamma_{p}^{1 / 2}}
$$

This is the growth rate for Cherenkov excitation of plasma waves (cf. Godfrey and Shanahan 1975; Egorenkov et al. 1983). We can estimate the importance of the Cherenkov excitation of plasma waves by evaluating the growth rate (7.4) for the set of fiducial parameters of a cold plasma and comparing it with the inverse of the dynamical time:

$$
\frac{\operatorname{Im}(\Delta)}{\gamma_{p} \Omega} \approx \frac{\lambda^{1 / 6}}{\gamma_{b} \gamma_{p}^{3 / 2}}\left(\frac{\omega_{B}}{\Omega}\right)^{1 / 2}=86\left(\frac{r}{R_{N S}}\right)^{-3 / 2} .
$$

From this, it follows that this instability may be important for $r \leqslant 20$. We shall see in Sec. 8 that the second criterion (5.5) is not satisfied for the Cherenkov excitation of Alfvén or $\mathrm{O}$ waves, so that the Cherenkov instability does not develop.

Similarly, we find the growth rate of the cyclotron excitation of transverse waves. Expanding in small $\Delta$, we find that the frequency shift near anomalous Doppler resonance is purely imaginary:

$$
\operatorname{Im}(\Delta)= \pm \frac{i \omega^{1 / 2} \omega_{B}^{1 / 2} \omega_{b}}{2 \gamma_{b} k c}=\frac{i \lambda^{1 / 2} \Omega}{\gamma_{p} \gamma_{b}^{1 / 2}}
$$

For the parameters of a cold plasma, the time scale implied by the growth rate (7.6) is much longer than the dynamical time everywhere inside the light cylinder:

$$
\frac{\operatorname{Im}(\Delta)}{\gamma_{p} \Omega} \approx \frac{\lambda^{1 / 2}}{\gamma_{p}^{2} \gamma_{b}^{1 / 2}}=10^{-4} \ll 1,
$$

which implies that hydrodynamic regime of the cyclotron instability is unimportant.

For propagation perpendicular to the magnetic field, the system exhibits magnetized Weibel instability (Weibel 1959). Expanding the determinant (7.1) for $\theta=\frac{1}{2} \pi$ near the upper-hybrid frequency $\omega=\left(\omega_{B}^{2}+2 \omega_{p}^{2}\right)^{1 / 2}+\Delta$, keeping terms up to second order in $\Delta$, we find the frequency shift

$$
\Delta=\frac{k \omega_{p}^{2}\left[\gamma_{b}^{1 / 2} k-\left(\gamma_{b}^{1 / 2} k^{2}-4 \omega_{b}^{2}\right)^{1 / 2}\right]}{2 \gamma_{b}^{1 / 2} \omega_{B}^{3}}
$$


which shows an instability for $k c<2 \omega_{b} / \gamma_{b}$, with a maximum growth rate

$$
\operatorname{Im}(\Delta)_{\max } \approx \frac{\omega_{p}^{2} \omega_{b}^{2}}{\gamma_{b}^{3 / 2} \omega_{B}^{3}},
$$

which is negligible for all reasonable pulsar plasma parameters.

\section{Oblique wave excitation in a cold plasma in the hydrodynamic regime}

In this section, we develop a general theory of hydrodynamic weak beam instabilities in a cold magnetized electron-positron plasma. We expand (7.1) in small $\omega_{b}$, keeping only the first terms. After some algebra, we obtain (Lyutikov 1998)

$$
K_{p}+\frac{A}{\hat{\omega}^{2}}+\frac{B}{\tilde{\omega}^{2}}=0
$$

where $K_{p}$ is the plasma part of the determinant (7.1) and the coefficients $A$ and $B$ may be found in Lyutikov (1998).

The term containing $1 / \hat{\omega^{2}}$ contributes to Cherenkov excitation, and the term containing $1 / \tilde{\omega}^{2}$ contributes to cyclotron excitation.

To find the growth rates, we expand the plasma part of (8.1) near the plasma modes $((4.1)$ and (4.4)) and the beam part near the resonances $\hat{\omega}=0$ (for Cherenkov excitation) or $\tilde{\omega}=0$ (for cyclotron instability). The expansion of the plasma part of (8.1) near the plasma modes is performed according to the relation

$$
\omega=\omega^{(0)}+\left.\Delta\left(\frac{\partial K_{p}}{\partial \omega}\right)\right|_{\omega^{(0)}},
$$

where $\omega^{(0)}$ are the solutions of $K_{p}=0$. The growth rates in a cold plasma are summarized in Table 9. For details of calculations, we refer the reader to Lyutikov (1998).

\section{Relativistic pair plasma: resonances}

From the low-frequency approximation to Alfvén-wave dispersion, we find that the possibility of Cherenkov excitation of Alfvén waves in a relativistically hot plasma depends on the parameter

$$
\mu_{h}=\frac{2 \gamma_{b} T_{p}^{1 / 2} \omega_{p}}{\omega_{B}}
$$

(cf. (6.3)). Numerically, $\mu_{h}$ and $\mu$ are equal for the chosen set of the fiducial numbers for the cold and hot cases.

Another limitation on possible resonance comes from the requirement that the waves in the plasma are not strongly damped at the location of the resonance. This is an important constraint on the resonance of the Alfvén wave, which is strongly damped at large wave vectors.

Using the dispersion relation for the Alfvén waves in the limit $k c \ll \omega_{p}$, we find that the cyclotron resonance on the Alfvén wave occurs at $k c \ll \omega_{p}$ for angles of propagation larger than

$$
\theta^{2}=\frac{\omega_{B} T_{p}^{1 / 2}}{\gamma_{b} \omega_{p}}
$$




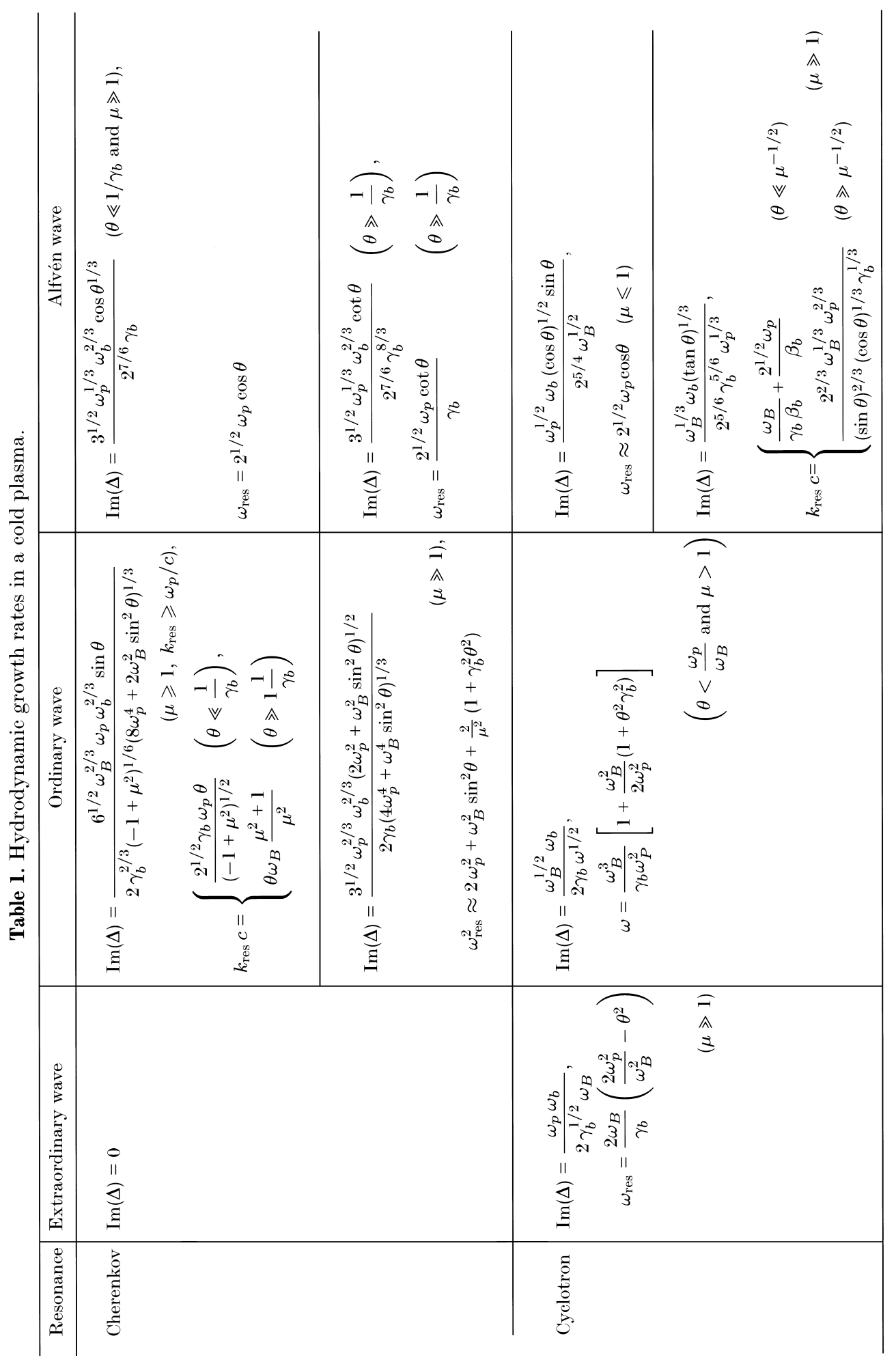


For smaller angles, the location of the cyclotron resonance on the Alfvén wave depends on the parameter

$$
\eta=\frac{\gamma_{b} \omega_{p}}{T_{p}^{3 / 2} \omega_{B}}
$$

If $\eta \ll 1$ (very hot plasma) then the cyclotron resonance on the Alfvén wave occurs in the region $\omega \gg \omega^{(0)}$, where Alfvén waves are strongly damped. If, on the other hand, $\eta \gg 1$ (warm plasma), the cyclotron resonance on the Alfvén wave occurs at approximately $\omega_{0}^{(h)}$, where Alfvén waves are not damped.

Since Alfvén waves cannot escape to infinity, they should be converted to electromagnetic modes before they are damped on the thermal particles. The Alfvén waves with large angles, which are generated with the frequency $\omega \ll \omega_{0}^{(h)}$, would have more time for the nonlinear processes to convert them into escaping modes than the Alfvén waves generated in a warm plasma with $\omega \approx \omega_{0}^{(h)}$ and small angles of propagation. Thus the cyclotron resonance on the Alfvén waves is likely to produce waves propagating in a cone around the magnetic field.

\section{Hydrodynamic wave excitation in relativistic pair plasma}

\subsection{Dielectric tensor for the beam-hot plasma system}

To simplify the analysis, we shall use the low-frequency approximation $\omega \ll \omega_{B}$ and the assumption of a very strong magnetic field $T_{p} \omega_{p}^{2} / \omega_{B}^{2} \ll 1$ from the very beginning. The components of the dielectric tensor are then given by

$$
\begin{aligned}
& \epsilon_{x x}=1+\frac{\omega_{p}^{2}}{\omega_{B}^{2}} T_{p}\left(1+n^{2} \beta_{T}^{2} \cos ^{2} \theta\right)-\frac{\omega_{b}^{2} \hat{\omega}^{2}}{\gamma_{b} \omega^{2} \tilde{\omega}^{2}}=\epsilon_{y y}, \\
& \epsilon_{x y}=\frac{-i \omega_{b}^{2} \omega_{B} \hat{\omega}}{\gamma_{b}^{2} \omega^{2} \tilde{\omega}^{2}}=-\epsilon_{y x}, \\
& \epsilon_{x z}=\frac{\omega_{p}^{2}}{\omega_{B}^{2}} T_{p} n^{2} \beta_{T}^{2} \cos \theta \sin \theta-\frac{k \omega_{b}^{2} \hat{\omega} \beta_{b} \sin \theta}{\gamma_{b} \omega^{2} \tilde{\omega}^{2}}=\epsilon_{z x}, \\
& \epsilon_{y z}=\frac{i k \omega_{b}^{2} \omega_{B} \beta_{b} \sin \theta}{\gamma_{b}^{2} \omega^{2} \tilde{\omega}^{2}}=-\epsilon_{z y}, \\
& \epsilon_{z z}=1-\frac{2 n^{2} \omega_{p}^{2}}{T_{p}\left(1-n^{2} \beta_{T}^{2} \cos ^{2} \theta\right)}+d T_{p} n^{2} \sin ^{2} \theta-\frac{\omega_{b}^{2}}{\gamma_{b}^{3} \hat{\omega}^{2}}-\frac{k^{2} \omega_{b}^{2} \beta_{b}^{2} \sin ^{2} \theta}{\gamma_{b} \omega^{2} \tilde{\omega}^{2}} .
\end{aligned}
$$

\subsection{Parallel propagation}

For parallel propagation, the growth rate for the Cherenkov excitation of plasma waves is

$$
\operatorname{Im}(\Delta)=\frac{3^{1 / 2} \omega_{p}^{1 / 3} \omega_{b}^{2 / 3}}{2^{7 / 6} \gamma_{b} T_{p}^{1 / 2}}=\frac{3^{1 / 2}\left(\Omega \omega_{B}\right)^{1 / 2} \lambda^{1 / 6}}{2^{2 / 3} \gamma_{b}\left(\gamma_{p} T_{p}\right)^{1 / 2}}
$$

(cf. Egorenkov et al. 1983).

Using the relations between the parameters of the hot plasma $((2.2)$ with $\langle\gamma\rangle=$ 
$\left.2 T_{p} \gamma_{p}\right)$, the condition of fast growth (5.4) for the growth rate (10.2) takes the form

$$
\frac{\operatorname{Im}(\Delta)}{\gamma_{p} \Omega} \approx \frac{\lambda^{1 / 6}}{\gamma_{b} \gamma_{p}^{3 / 2} T_{p}^{1 / 2}}\left(\frac{\omega_{B}}{\Omega}\right)^{1 / 2}=20\left(\frac{r}{R_{N S}}\right)^{-3 / 2}
$$

For fixed values of $\gamma_{b}$ and $\gamma_{p}$, the growth rate for the Cherenkov excitation of plasma waves in a hot plasma is smaller by a factor $T_{p}^{2 / 3}$ compared with that for a cold plasma.

The growth rate for the cyclotron excitation of transverse waves is

$$
\Delta=i \frac{T_{p}^{1 / 2} \omega_{p} \omega_{b}}{2 \gamma_{b}^{1 / 2} \omega_{B}}=\left(\frac{\lambda T_{p}}{\gamma_{b}}\right) \frac{\Omega}{\gamma_{p}} .
$$

Comparison of this growth rate with the inverse of the dynamical time gives

$$
\frac{\operatorname{Im}(\Delta)}{\Omega \gamma_{p}}=\frac{1}{\gamma_{p}^{2}}\left(\frac{\lambda T_{p}}{\gamma_{b}}\right)^{1 / 2} \approx \frac{1}{\gamma_{p}^{2}}\left(\frac{r}{R_{N S}}\right)^{3 / 2}=10^{-4}\left(\frac{r}{R_{N S}}\right)^{3 / 2}<1
$$

It follows from (10.5) and (7.7) if follows that cyclotron excitation of the transverse waves in the hydrodynamic regime is not affected by the relativistic temperature of the plasma particles, and is not important in the pulsar magnetosphere.

Similarly to the cold case, we omit the details of the calculations of the growth rates (see Lyutikov 1998), and conclude this section with a table of hydrodynamic growth rates in a relativistically hot pair plasma (Table 11).

\section{Kinetic instabilities}

As we have discussed in Sec. 5, a general beam instability may be treated analytically in the hydrodynamic and kinetic limiting cases. We have considered hydrodynamic beam instabilities in a pair plasma in Secs 8 and 10. Now we turn to the kinetic regime of instabilities. The condition for the kinetic consideration to apply is the opposite of the condition (5.2). It requires a substantial scatter in the velocities of the resonant particles. In what follows, we assume that the distribution of the beam particles is described by the relativistic, one-dimensional Maxwellian distribution:

$$
f\left(p_{z}\right)=n_{b} \frac{1}{2 K_{1}\left(1 / T_{b}\right) \gamma_{b}} \exp \left(-\frac{p_{\mu} U^{\mu}}{T_{b}}\right),
$$

where $n_{b}$ is the density of the beam measured in the laboratory frame (the Lorentzinvariant proper density is $\left.n_{b} \gamma_{b}\right), U^{\mu}=\left(\gamma_{b}, \boldsymbol{\beta}_{b} \gamma_{b}\right)$ is the four-velocity of the rest frame of the beam, $T_{b}$ is the beam temperature in units of $m c^{2}$, and $K_{1}$ is a modified Bessel function.

This distribution function may be simplified in the limit of a cold beam (in its frame), $T_{b} \ll 1$, and large streaming velocity $\gamma_{b} \gg 1$. We then find

$$
f\left(p_{z}\right)=\frac{n_{b}}{(2 \pi)^{1 / 2}} \exp \left(-\frac{\left(p_{z}-p_{b}\right)^{2}}{2 p_{t}^{2}}\right),
$$

where $p_{t}^{2}=\gamma_{b}^{2} T_{b} m c$ is the scatter in parallel moments. 


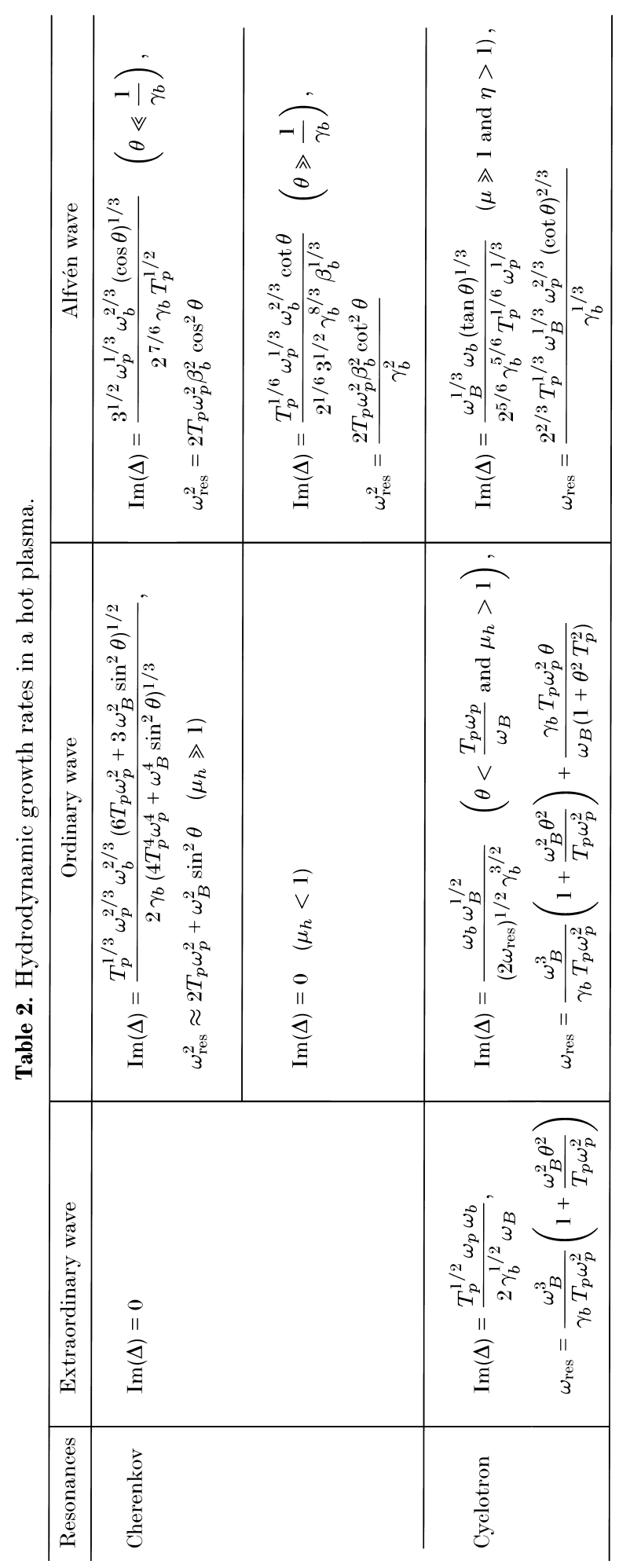


In the case of kinetic instability the growth rate is given by (see e.g. Melrose 1980)

$$
\Gamma=-\left.\frac{\left(e_{\alpha}^{*} \epsilon_{\alpha \beta}^{\prime \prime} e_{\beta}\right)}{\frac{1}{\omega^{2}} \frac{\partial}{\partial \omega} \omega^{2}\left(e_{\alpha}^{*} \epsilon_{\alpha \beta}^{\prime} e_{\beta}\right)}\right|_{\omega=\omega(\mathbf{k})}
$$

where $\epsilon_{\alpha \beta}^{\prime}$ and $\epsilon_{\alpha \beta}^{\prime \prime}$ are the Hermitian and anti-Hermitian parts of the dielectric tensor, $\omega(\mathbf{k})$ is the frequency of the excited normal modes of the medium, and $e_{\alpha}$ is its polarization vector. The anti-Hermitian parts of the dielectric tensor are due to the resonant interaction of the particles from the beam at Cherenkov (6.1) and cyclotron (6.2) resonances. Using the polarization vectors (4.12) and (4.13), we find that for the quasitransverse waves $\left(\mathrm{O}\right.$ mode $\omega \gg \omega_{0}^{(h)}$, Alfvén mode $\omega \ll \omega_{0}^{(h)}$ and O mode $\left.\omega \approx \omega_{0}^{(h)}\right), \theta \gg \omega_{B}^{2} / T_{p} \omega_{p}^{2}$, while for the $\mathrm{O}$ mode at the crossover point and $\theta \ll \omega_{B}^{2} / T_{p} \omega_{p}^{2}$

$$
\frac{1}{\omega^{2}} \frac{\partial}{\partial \omega} \omega^{2}\left(\mathbf{e} \cdot \boldsymbol{\epsilon}^{\prime} \cdot \mathbf{e}\right)= \begin{cases}\frac{2}{\omega} & \text { (cold plasma) }, \\ \frac{T_{p} \omega}{\omega_{p}^{2}} & \text { (hot plasma) }\end{cases}
$$

With polarization vectors (4.6) and (4.8) we find from, that for the quasitransverse parts of the waves,

$$
\begin{aligned}
\mathbf{e}_{X} \cdot \boldsymbol{\epsilon}^{\prime \prime} \cdot \mathbf{e}_{X}= & -i \frac{2 \pi^{2} e^{2}}{\omega^{2} m} \int \frac{d p_{z}}{\gamma} \hat{\omega} f\left(p_{z}\right) \delta\left(\hat{\omega}-\frac{\omega_{B}}{\gamma}\right), \\
\mathbf{e}_{O} \cdot \boldsymbol{\epsilon}^{\prime \prime} \cdot \mathbf{e}_{O}= & \frac{4 \pi^{2} e^{2}}{m \omega} \int d p_{z} v_{z} \frac{\partial f\left(p_{z}\right)}{\partial p_{z}} \delta(\hat{\omega}) \sin ^{2} \theta \\
& +\frac{2 \pi^{2} e^{2}}{\omega^{2} \omega_{B} m} \int d p_{z}\left(k v_{z}-\omega \cos \theta\right)^{2} f\left(p_{z}\right) \delta\left(\hat{\omega}-\frac{\omega_{B}}{\gamma}\right) \\
= & \epsilon_{O}^{\prime \prime C h}+\epsilon_{O}^{\prime \prime C}, \\
\mathbf{e}_{A} \cdot \boldsymbol{\epsilon}^{\prime \prime} \cdot \mathbf{e}_{A}= & \frac{\pi^{2} e^{2}}{m \omega} \frac{\omega^{4}}{\omega_{p}^{4}} \int d p_{z} v_{z} \frac{\partial f\left(p_{z}\right)}{\partial p_{z}} \delta(\hat{\omega}) \tan ^{2} \theta \\
& +\frac{2 \pi^{2} e^{2}}{\omega^{2} \omega_{B} m} \int d p_{z}\left(\omega-k v_{z} \cos \theta\right)^{2} f\left(p_{z}\right) \delta\left(\hat{\omega}-\frac{\omega_{B}}{\gamma}\right) \\
= & \epsilon_{A}^{\prime \prime C h}+\epsilon_{A}^{\prime \prime C},
\end{aligned}
$$

where we have split the anti-Hermitian part for the $\mathrm{O}$ and Alfvén modes into two parts: $\epsilon^{\prime \prime C h}$ is due to Cherenkov resonance and $\epsilon^{\prime \prime C}$ is due to cyclotron resonance.

The relation (11.7) is valid are valid for both cold and hot plasmas, except that in the latter, the Cherenkov-resonance term is replaced by

$$
\epsilon_{A}^{\prime \prime C h(h)} \equiv\left(\mathbf{e}_{A} \cdot \epsilon^{\prime \prime} \cdot \mathbf{e}_{A}\right)^{(h)}=\frac{\pi^{2} e^{2}}{m \omega} \frac{\omega^{4}}{T_{p}^{2} \omega_{p}^{4}} \int d p_{z} v_{z} \frac{\partial f\left(p_{z}\right)}{\partial p_{z}} \delta(\hat{\omega}) \tan ^{2} \theta
$$

For Cherenkov excitation of the $\mathrm{O}$ mode in the limit $\mu_{h} \gg 1$ (when the resonance 
occurs at the crossover point), we find

$$
\left(\mathbf{e}_{O} \cdot \boldsymbol{\epsilon}^{\prime \prime} \cdot \mathbf{e}_{O}\right)_{C h}= \begin{cases}\frac{4 \pi^{2} e^{2}}{m \omega} \int d p_{z} v_{z} \frac{\partial f\left(p_{z}\right)}{\partial p_{z}} \delta(\hat{\omega}) & \left(\theta \ll \frac{2 \omega_{p}^{2}}{\omega_{B}^{2}}\right), \\ \frac{4 \pi^{2} e^{2}}{m \omega} \frac{\omega_{0}^{4}}{\omega_{B}^{4} \cos ^{2} \theta \sin ^{2} \theta} \int d p_{z} v_{z} \frac{\partial f\left(p_{z}\right)}{\partial p_{z}} \delta(\hat{\omega}) & \left(\theta \gg \frac{2 \omega_{p}^{2}}{\omega_{B}^{2}}\right) .\end{cases}
$$

\subsection{Parallel propagation}

We first consider the important, separate case of parallel propagation.

Using the polarization vectors $\mathbf{e}_{l}=(0,0,1)$ for longitudinal waves and $\mathbf{e}_{t}=(1,0,0)$ for transverse waves, we find growth rates

$$
\begin{aligned}
\Gamma_{t} & =\frac{\pi \omega_{p, r e s}^{2}}{4 \omega}(f)_{\mathrm{res}} \approx \frac{\pi \omega_{p, r e s}^{2}}{\omega \Delta \gamma}, \\
\omega & =\frac{\omega_{B}^{3}}{\gamma_{b} T_{p} \omega_{p}^{2}} \\
\Gamma_{l} & =\frac{\pi \omega_{p}^{2} \omega_{p, r e s}^{2}}{T_{p} k c \omega^{2}}\left(\gamma^{3} \frac{\partial f}{\partial \gamma}\right)_{\mathrm{res}} \approx \frac{n_{b}}{n_{p}} \frac{\pi \omega_{p} \gamma_{b}^{3}}{T_{p}^{5 / 2} \Delta \gamma^{2}}, \\
\omega & =\omega_{0}=\left(2 T_{p}\right)^{1 / 2} \omega_{p}
\end{aligned}
$$

(Kazbegi et al. 1991).

The kinetic growth rates (11.10a) and (11.11a) can be compared with the growth rates in the hydrodynamic regime, (7.4) and (7.6). In the hydrodynamic regime, both cyclotron and Cherenkov growth rates are proportional to negative powers of the particle's Lorentz factor. This is a significant factor for the primary beam and for the particles from the tail of the plasma distribution. In contrast, the kinetic growth rates (11.10a) and (11.11a) are not suppressed by relativistic streaming of resonant particles. On the other hand, they scale linearly with a small ratio of the beam density to plasma density, while the hydrodynamic growth rates (7.4) and (7.6) are proportional to the $\frac{1}{3}$ and $\frac{1}{2}$ powers of this ratio.

The kinetic growth rates for oblique propagation are summarized in Table 12.1.

\section{Hydrodynamic versus kinetic instabilities}

Having calculated the growth rates for the hydrodynamic and kinetic regimes of the Cherenkov and cyclotron instabilities, we can check whether the conditions for the corresponding regimes are satisfied.

\subsection{Cherenkov resonance}

The condition for the hydrodynamic regime for Cherenkov excitation is given by (5.3) (the condition for the kinetic regime is reversed). When the scatter in the velocity of the resonant particles is due to scatter in the parallel velocity (and not to the scatter in the pitch angles) the condition (5.3) with the parallel growth rate $(10.2)$ gives the following requirement for hydrodynamic-type Cherenkov instability:

$$
\frac{\gamma_{b}^{2}}{T_{p}^{1 / 2} \Delta \gamma \lambda^{1 / 3}} \gg 1,
$$




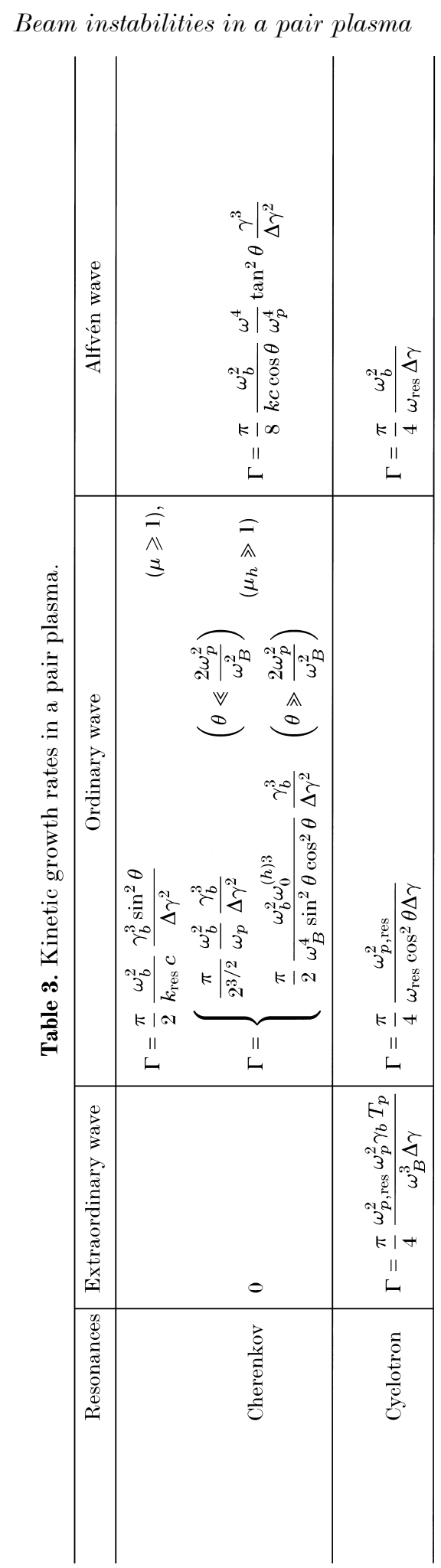


which is well satisfied for the chosen plasma parameters. This implies that if the primary beam does not acquire any significant transverse gyrational energy as it propagates outwards in the pulsar magnetosphere then Cherenkov-type instabilities occur in the hydrodynamic regime.

\subsection{Cyclotron resonance}

For cyclotron resonance, the left-hand side of (5.3) is dominated by the last term. For cyclotron excitation of the $\mathrm{X}$-mode, the condition (5.3) with the growth rate (7.6), for the hydrodynamic-type instability to apply,

$$
\Delta \gamma \ll\left(T_{p} \lambda\right)^{1 / 2} \frac{\gamma_{b}^{3 / 2} \Omega}{\gamma_{p} \omega_{B}}=10^{-7}\left(\frac{r}{R_{N S}}\right)^{3},
$$

which is most probably not satisfied even in the outer regions of the pulsar magnetosphere.

The condition for the kinetic approximation to hold for the cyclotron excitation of the $\mathrm{X}$ mode follows from the reverse of (5.3) and Table 12.1:

$$
\Delta \gamma \gg\left(\frac{\gamma_{\mathrm{res}}^{3} \lambda \lambda_{\mathrm{res}} T_{p}}{\gamma_{p}}\right)^{1 / 2} \frac{\Omega}{\omega_{B}},
$$

which is well satisfied inside the pulsar magnetosphere.

We conclude from these estimates that the cyclotron instability in the pulsar magnetosphere occurs in the kinetic regime. This is different from the electrostatic Cherenkov instabilities on the primary beam, which occur in the hydrodynamic regime.

This difference is very important for theories of pulsar radio emission. Kinetic instabilities, in contrast to hydrodynamic ones, are not suppressed by the large relativistic factor of the resonant particles. Thus the kinetic instabilities are more favourable as a possible source of the pulsar radio emission.

It is possible to illustrate graphically the difference between the hydrodynamic regime of the Cherenkov instability and the kinetic regime of the cyclotron instability. On the frequency-wave-vector diagram for the $\mathrm{O}$ mode (Fig. 3), the dispersion curves of the cyclotron wave in the beam $\omega=k v_{b} \cos \theta-\omega_{B} / \gamma_{b}$ are almost parallel to the dispersion curves of the excited waves in the plasma at the location of the resonance. Thus a small change in the velocity of the resonant particles results in a considerable change in the resonant frequency. This vindicates the kinetic approximation, which requires a large bandwidth of the growing waves. In contrast, for the very large streaming $\gamma$ factor of the primary beam (so that $\mu, \mu_{h} \gg 1$ ), the Cherenkov resonances on the $\mathrm{O}$ and $\mathrm{X}$ modes occur approximately at the crossover frequency in a narrow frequency band.

\section{Conclusions}

In this paper, we have considered normal modes and wave excitation in the strongly magnetized electron-positron plasma of a pulsar magnetosphere. We have found the locations of resonances, and have calculated the growth rates for Cherenkov and cyclotron excitation of $\mathrm{O}, \mathrm{X}$ and Alfvén waves in two limiting regimes of hydrodynamic and kinetic instabilities, taking into account the angular dependence of the growth rates. The main results of this paper are as follows: 
(i) Cherenkov instabilities develop in the hydrodynamic regime while cyclotron instabilities develop in the kinetic regime.

(ii) Cherenkov instability on the primary beam may develop on Alfvén waves in the regions close to the stellar surface and on the $\mathrm{O}$ mode in the outer regions of the pulsar magnetosphere.

(iii) Cyclotron instability can develop on all three wave branches. On the Alfvén branch, it does not develop in a very hot plasma.

(iv) The typical ranges of angles (in the plasma frame) with the highest growth rates are

$$
\begin{array}{ll}
\delta \theta \approx \omega_{p}^{2} / \omega_{B}^{2} & \text { for Cherenkov excitation of the } \mathrm{O} \text { mode; } \\
\delta \theta \approx 1 / \gamma_{b} & \text { for Cherenkov excitation of the Alfvén mode; } \\
\delta \theta \approx \omega_{p} / \omega_{B} & \text { for cyclotron excitation of the } \mathrm{O} \text { and } \mathrm{X} \text { modes; } \\
\delta \theta \approx 1 & \text { for cyclotron excitation of the Alfvén mode; }
\end{array}
$$

(v) We also note that Cherenkov instability due to the relative drift of the plasma particles can develop only on the Alfvén mode. This due to the fact that in the inner magnetosphere, where the instability due to the relative drift of the plasma particles can develop, the approximation $\omega_{B}=\infty$ is possible. In this approximation, the $\mathrm{O}$ mode is always superluminal, and cannot be excited by Cherenkov resonance.

These arguments suggest that electromagnetic cyclotron instabilities are more likely to develop in the pulsar magnetosphere than electrostatic ones.

\section{Acknowledgements}

I should like to thank Roger Blandford for his support and comments, George Machabeli and George Melikidze for useful cooperation, and Abastumani Astrophysics Observatory for hospitality during my stays in Tbilisi. This research was supported by the NSF under Grant AST-9529170 and by the CITA Fellowship.

\section{References}

Arons, J. 1981 In: Proceedings of Varenna Summer School and Workshop on Plasma Astrophysics (ed. T. Guyenne and G. Levy), p. 273. ESA, Noordwijk, The Netherlands.

Arons, J. 1983 Astrophys. J. 266, 215.

Arons, J. and Barnard, J. J. 1986 Astrophys. J. 302, 120.

Asseo, E. 1995 Mon. Not. R. Astron. Soc. 276, 74.

Asseo, E., Pellat R. and Sol H. 1983 Astrophys. J. 266, 201.

Beskin, V.S. Gurevich, A. V. and Istomin Y. N. 1983 Soviet Phys. JETP 85, 234.

Daugherty, J. K. and Harding, A. K. 1983 Astrophys. J. 273, 761.

Egorenkov, V. D., Lominadze, D. G. and Mamredze, P. G. 1983 Astrofizika, 19, 753.

Godfrey, B. B. and Shanahan, W. R. 1974 IEEE Trans. Plasma. Sci. P53, 60.

Godfrey, B. B. and Shanahan, W. R. 1975 Phys. Fluids 18, 346.

Goldreich, P. and Julian W. H. 1969 Astrophys. J. 157, 869.

Goldreich, P. and Keeley D. A. 1971 Astrophys. J. 170, 463.

Kazbegi, A. Z., Machabeli, G.Z. and Melikidze, G. I. 1991 Mon. Not. R. Astron. Soc. 253, 377.

Lyutikov, M. 1998 physics/9807022.

Lyutikov, M., Blandford, R. D. and Machabeli, G.Z. 1999a Astrophys. J. 512, 804. 
Lyutikov, M., Machabeli G. Z. and Blandford R. D. 1999b Mon. Not. R. Astron. Soc. 305, 338. Melrose, D. B. 1980 Plasma Astrophysics: Nonthermal Processes in Diffuse Magnetized Plasmas, Vol. I. Gordon and Breach, New York.

Melrose, D. B. 1995 J. Astron. Astrophys. 16, 137.

Melrose, D. B. 1982 Aust. J. Phys. 35, 41.

Silin, V. P. 1960 Soviet Phys. JETP 11, 1136.

Sturrock, P. A. 1960 J. Appl. Phys. 31, 2052.

Suvorov, E. V. and Chugunov, Yu. V. 1975 Astrofizika 11, 305.

Tademaru, E. 1973, Astrophys. J. 183, 625.

Tsytovich, V. N. and Kaplan, S. A. 1972 Astrofizika 8, 411.

Volokitin, A. S., Krasnosel'skikh, V. V. and Machabeli, G. Z. 1985 Soviet J. Plasma Phys. 11, 310

Weibel, E. 1959 Phys. Rev. Lett. 2, 83.

Zank, G. P. and Greaves, R. G. 1995 Phys. Rev. E51, 6079. 